\title{
PRIORITIZATION METHOD FOR FRONTIER DMUs: A DISTANCE-BASED APPROACH
}

\author{
ALIREZA AMIRTEIMOORI, GHOLAMREZA JAHANSHAHLOO, \\ AND SOHRAB KORDROSTAMI
}

Received 7 October 2003 and in revised form 27 May 2004

In nonparametric methods, if the number of observations is relatively small as compared to the sum of number of inputs and outputs, many units are evaluated as efficient. Several methods for prioritizing these efficient units are reported in literature. Andersen et al. and Mehrabian et al. proposed two methods for ranking efficient units, but both methods break down in some cases. This paper describes a new DEA ranking approach that uses $L_{2}$-norm.

\section{Introduction}

Data envelopment analysis (DEA) originally from Farell's [7] seminal work and popularized by Charnes et al. [5] has gained a wide range of applications measuring comparative efficiency. The main point of DEA is to assign an efficiency score to each of the decisionmaking units (DMUs). This efficiency score depends on the orientation of the problem. The data set in DEA that consists of $n$ DMUs defines a production set with a subset consisting of boundary points that form an efficient frontier. The main object of DEA is to determine the relative position of a unit in a production set. Banker et al. [3] and Charnes, Cooper, and Rhodes (CCR) [5] used the term Production possibility set (PPS) for production set. In formal terms, we define $T$ as

$$
T=\{(X, Y) \mid \text { input } X \text { can produce output } Y\}
$$

The usefulness of the set notion for our purposes comes from the fact that it brings along two further notions, namely, (i) that of the boundary (or frontier) of the set, and (ii) that of the interior of the set. This permits one to distinguish between DMUs that belong to the interior of the production set, which are called inefficient, and those that do belong to a particular part of the frontier and are called efficient. DEA assigns 1 to all efficient DMUs. This approach ranks all DMUs based upon their efficiency score. In cases where several DMUs have the same efficiency score 1, DEA approach cannot rank these 
DMUs. However, decision makers are often interested in a complete ranking, beyond the dichotomized classification, in order to refine the evaluation of the units. Discriminating between these efficient DMUs is an interesting subject. This problem has recently been studied by a number of DEA researchers (see $[1,2,6,8]$ etc.). Andersen and Petersen [1] proposed a method for ranking efficient DMUs (AP model). In their method the column corresponding to the unit under consideration is omitted from technological matrix. However, the feasibility for the problem is not guaranteed and for small variation in data, some problems are not stable. Another method was suggested by Mehrabian et al. [8] (MAJ model). Although in MAJ model the basic idea is same as AP model, the movement strategy towards frontier is different from AP model. However, in some cases, the infeasibility for the problem may occur; we will prove that too. The method proposed by the authors removes the existing difficulties in both methods. The paper is structured as follows. Section 2 provides basic DEA models. An introduction to AP and MAJ models is given in Section 3. The next section of the paper addresses a method for ranking efficient DMUs. Some numerical examples are solved in Section 5. Conclusions appear in Section 6.

\section{Background}

To describe the DEA efficiency measurement, let there be $n$ DMUs and let the performance of each DMU be characterized by a production process of $m$ inputs $\left(x_{i j}, i=\right.$ $1, \ldots, m)$ to yield $s$ outputs $\left(y_{r j}, r=1, \ldots, s\right)$.

Definition 2.1. $\mathrm{DMU}_{p}$ is called inefficient if and only if it is dominated within the set of the $n$ DMUs.

The $p$ th DMU is dominated when

$$
\begin{aligned}
\sum_{j=1}^{n} \lambda_{j} x_{i j} \leq x_{i p} & \text { for } i=1,2, \ldots, m, \\
\sum_{j=1}^{n} \lambda_{j} y_{r j} \geq y_{r p} & \text { for } r=1,2, \ldots, s \\
\lambda_{j} \geq 0, & \text { for } j=1,2, \ldots, n,
\end{aligned}
$$

and at least one of the above conditions is a strict inequality. Every nondominant DMU is called efficient. To estimate a DEA efficiency score of the specific $p$ th DMU, we use the following original DEA model (Charnes et al. [5]):

$$
\begin{gathered}
\text { Maxe } e_{p} \quad \text { subject to } e_{q}=\frac{\sum_{r=1}^{s} u_{r} y_{r q}}{\sum_{i=1}^{m} v_{i} x_{i q}}, \quad q=1, \ldots, n, \\
0 \leq e_{q} \leq 1, \quad q=1, \ldots, n \\
u_{r} \geq \epsilon, \quad r=1, \ldots, s \\
v_{i} \geq \epsilon, \quad i=1, \ldots, m,
\end{gathered}
$$


where $e_{p}, p=1, \ldots, n$, are the efficiency of $\mathrm{DMU}_{p}$. This model can be reduced to a nonratio format in the usual manner of Charnes and Cooper [4] as follows:

$$
\begin{gathered}
\operatorname{Max} \bar{e}_{p}=\sum_{r=1}^{s} u_{r} y_{r p}, \quad \text { subject to } \sum_{i=1}^{m} v_{i} x_{i p}=1, \\
\sum_{r=1}^{s} u_{r} y_{r j}-\sum_{i=1}^{m} v_{i} x_{i j} \leq 0, \quad j=1, \ldots, n, \\
u_{r} \geq \epsilon, \quad r=1, \ldots, s, \\
v_{i} \geq \epsilon, \quad i=1, \ldots, m .
\end{gathered}
$$

Hence, to determine all DMU efficiencies, the above program is solved $n$ times, once for each $\mathrm{DMU}_{p}, p=1, \ldots, n$.

\section{AP model and MAJ model}

3.1. AP model. Andersen and Petersen [1] developed a procedure for ranking efficient units. Their method enables an efficient $\mathrm{DMU}_{p}$ to achieve an efficiency score greater than or equal to one by removing the $p$ th constraint in (2.3). The mathematical formulation of this model is as follows:

$$
\begin{gathered}
\operatorname{Max} \sum_{r=1}^{s} u_{r} y_{r p}, \quad \text { subject to } \sum_{i=1}^{m} v_{i} x_{i p}=1, \\
\sum_{r=1}^{s} u_{r} y_{r j}-\sum_{i=1}^{m} v_{i} x_{i j} \leq 0, \quad j=1, \ldots, n, j \neq p, \\
u_{r} \geq \epsilon, \quad r=1, \ldots, s, \\
v_{i} \geq \epsilon, \quad i=1, \ldots, m .
\end{gathered}
$$

The dual formulation of (3.1) is as follows:

$$
\begin{gathered}
\operatorname{Min} \theta_{p}-\epsilon\left[\sum_{i=1}^{m} s_{i}^{-}+\sum_{r=1}^{s} s_{r}^{+}\right] \text {subject to } \sum_{\substack{j=1 \\
j \neq p}}^{n} \lambda_{j} x_{i j}+s_{i}^{-}=\theta_{p} x_{i p}, \quad i=1, \ldots, m, \\
\sum_{\substack{j=1 \\
j \neq p}}^{n} \lambda_{j} y_{r j}-s_{r}^{+}=y_{r p}, \quad r=1, \ldots, s, \\
\lambda_{j}, s_{i}^{-}, s_{r}^{+} \geq 0, \quad \forall i, j, r .
\end{gathered}
$$

However, there are some problematic areas with this methodology that is shown by the following example. There are 5 DMUs with two inputs and two outputs. We consider three cases of DMU 1, denoted by DMU $1_{1}, \mathrm{DMU}_{2}$ and DMU $1_{3}$ (see Table 3.1).

The AP model for evaluating DMU $1_{2}$ is infeasible, this model for evaluating DMU $1_{3}$ leads to a large score. Table 3.2 presents the results. 
Table 3.1. The data set for infeasibility and unstability of AP model.

\begin{tabular}{cccccccc}
\hline & DMU $1_{1}$ & DMU $1_{2}$ & DMU $1_{3}$ & DMU2 & DMU3 & DMU 4 & DMU 5 \\
\hline$I_{1}$ & 2 & 0 & 0.09 & 5 & 10 & 10 & 2 \\
$I_{2}$ & 10 & 10 & 10 & 7 & 5 & 7 & 13 \\
$O_{1}$ & 2 & 2 & 2 & 2 & 3 & 2 & 3 \\
$O_{2}$ & 3 & 3 & 3 & 3 & 1 & 1 & 2 \\
\hline
\end{tabular}

Table 3.2. The AP and CCR efficiency indexes.

\begin{tabular}{cccc}
\hline Efficiency index & DMU $1_{1}$ & ${\text { DMU } 1_{2}}$ & ${\text { DMU } 1_{3}}$ \\
\hline CCR & 1.000 & 1.000 & 1.000 \\
AP & 1.700 & Infeasible & 33.300 \\
\hline
\end{tabular}

3.2. MAJ model. An alternative approach suggested in the literature, is MAJ model (Mehrabian and et al. [8]). The mathematical formulation of MAJ model is as follows:

$$
\begin{gathered}
\text { Min } 1+W_{p} \quad \text { subject to } \sum_{\substack{j=1 \\
j \neq p}}^{n} \lambda_{j} x_{i j}+s_{i}^{-}=W_{p}+x_{i p}, \quad i=1, \ldots, m, l \\
\sum_{\substack{j=1 \\
j \neq p}}^{n} \lambda_{j} y_{r j}-s_{r}^{+}=y_{r p}, \quad r=1, \ldots, s, \\
\lambda_{j}, s_{i}^{-}, s_{r}^{+} \geq 0, \quad \forall i, j, r .
\end{gathered}
$$

In what follows, we show that MAJ model fails to rank DMUs in some cases.

(i) First deficiency. For special data, MAJ model is infeasible. To show this, consider the dual formulation of MAJ model as follows:

$$
\begin{gathered}
\operatorname{Max} \sum_{r=1}^{s} u_{r} y_{r p}-\sum_{i=1}^{m} v_{i} x_{i p}, \quad \text { subject to } \sum_{r=1}^{s} u_{r} y_{r j}-\sum_{i=1}^{m} v_{i} x_{i j} \leq 0, \quad j=1, \ldots, n, j \neq p, \\
\sum_{i=1}^{m} v_{i} x_{i p}=1, \\
u_{r}, v_{i} \geq 0, \quad \forall i, r .
\end{gathered}
$$

Consider the system of linear equations

$$
Y^{p} d_{u}=0, \quad Y_{p} d_{u}-t=0,
$$


Table 3.3. The data set.

\begin{tabular}{cccccc}
\hline & DMU 1 & DMU2 & DMU3 & DMU 4 & DMU 5 \\
\hline$I_{1}$ & 2 & 0 & 0.1 & 5 & 10 \\
$I_{2}$ & 8 & 8 & 8 & 5 & 4 \\
$O_{1}$ & 1 & 1 & 1 & 1 & 2 \\
$O_{2}$ & 2 & 2 & 2 & 1 & 1 \\
\hline
\end{tabular}

Table 3.4. The results for MAJ model.

\begin{tabular}{cccccc}
\hline & DMU 1 & DMU2 & DMU 3 & DMU 4 & DMU 5 \\
\hline MAJ input orientation & 4 & 2 & 3 & 6 & 1 \\
MAJ output orientation & 4 & 1 & 3 & 6 & 2 \\
\hline
\end{tabular}

where $t>0 ; Y^{p}$ is an $(n-1) s$-matrix of outputs of $\mathrm{DMU}_{1}, \ldots, \mathrm{DMU}_{p-1}, \mathrm{DMU}_{p+1}, \ldots$, $\mathrm{DMU}_{n} ; Y_{p}$ is the output vector of $\mathrm{DMU}_{p}$; and $d_{u} \geq 0$ is a nonzero $s$-vector. For some $Y^{p}$ and $Y_{p}$, (3.5) has a solution. For example

$$
\begin{gathered}
Y_{p}=\left(0, \ldots, 0, y_{k p}>0,0, \ldots, 0\right), \\
Y^{p}=\left(0, \ldots, 0, \frac{t}{y_{k p}}, 0, \ldots, 0\right), \\
d_{u}=\left[\begin{array}{cccccc}
y_{11} & y_{12} & \ldots & y_{1 p}=0 & \ldots & y_{1 s} \\
y_{21} & y_{22} & \ldots & y_{2 p}=0 & \ldots & y_{2 s} \\
\vdots & \vdots & \ddots & \vdots & \vdots & \vdots \\
y_{n-1,1} & y_{n-1,2} & \cdots & y_{n-1, p}=0 & \cdots & y_{n-1, s}
\end{array}\right]
\end{gathered}
$$

is a solution to (3.5). Now, consider $d=\left[d_{u}, 0\right]^{T}$. It can be seen that $A d=0, d \geq 0, d \neq 0$, $C d>0$, where $A$ is the technological matrix and $C$ is the cost coefficient in the objective function in (3.4). This means that for special data, (3.4) is unbounded and by duality theorem, MAJ model is infeasible. This completes the proof.

(ii) Second deficiency. The following example shows that in some cases of MAJ model, ranking in input orientation is different from ranking in output orientation. Our illustrative example entails 5 DMUs (see Table 3.3). Each unit consumes two inputs to produce two outputs.

Running MAJ model yields the results presented in Table 3.4.

It is evident that ranking in input orientation is different from ranking in output orientation (see DMU2 and DMU5). 
Table 4.1. The data set for the simple example.

\begin{tabular}{cccc}
\hline & DMU 1 & DMU2 & DMU3 \\
\hline$I_{1}$ & 5 & 2 & 1 \\
$I_{2}$ & 1 & 2 & 4 \\
$O$ & 1 & 1 & 1 \\
\hline
\end{tabular}

\section{A distance-based approach}

This section describes a new DEA-based ranking approach that uses $L_{2}$-norm. In the proposed method, to rank an efficient unit, this unit is omitted from PPS and the new frontier is constructed. Then, the shortest distance $\left(L_{2}\right.$-norm) from this unit to the new frontier is determined. This is done for all DEA efficient units.

Definition 4.1. A surface $H=\left\{(X, Y):-\alpha^{T} X+\beta^{T} Y=0, \alpha \geq 0, \beta \geq 0\right\} \cap T_{c}$ is called a supporting efficient surface if for each observation $j=1, \ldots, n,-\alpha^{T} X_{j}+\beta^{T} Y_{j} \leq 0$ and at least $m+s-1$ inequalities are binding.

To have at least $m+s-1$ inequalities $-\alpha^{T} X_{j}+\beta^{T} Y_{j} \leq 0$ as equalities, we use the slack variables $s_{j}$. The constraints $s_{j} \leq\left(1-\gamma_{j}\right) M, s_{j} \geq 0, \gamma_{j} \in\{0,1\}, \sum_{j \in E} \gamma_{j} \geq m+s-1$ force some of the $s_{j}$ at zero level ( $M$ is a large positive constant). Clearly, selecting $\gamma_{t}=1$ forces the $s_{t}=0$. ( $E$ denotes the set of all CCR-efficient DMUs.) Consider the following program:

$$
\begin{gathered}
D_{p}=\operatorname{Max}-\alpha^{T} X_{p}+\beta^{T} Y_{p} \quad \begin{array}{c}
\text { subject to }-\alpha^{T} X_{j}+\beta^{T} Y_{j}+s_{j}=0, \quad j \in E, j \neq p, \\
\alpha^{T} 1_{m}+\beta^{T} 1_{s}=1,
\end{array} \\
s_{j} \leq\left(1-\gamma_{j}\right) M, \quad j \in E, j \neq p, \\
\sum_{j \in E, j \neq p} \gamma_{j} \geq m+s-1, \\
\alpha \geq \epsilon \cdot 1_{m}, \\
\beta \geq \epsilon \cdot 1_{s}, \\
\gamma_{j} \in\{0,1\}, \quad j \in E, j \neq p, \\
s_{j} \geq 0, \quad j \in E, j \neq p,
\end{gathered}
$$

where $\alpha$ is an $m$-vector, $\beta$ is an $s$-vector, $\epsilon>0, X_{j}=\left(x_{1 j}, \ldots, x_{m j}\right)^{T}, Y_{j}=\left(y_{1 j}, \ldots, y_{s j}\right)^{T}$ and $1 .=(1,1, \ldots, 1)^{T}$. It is inherent in this formulation of the DEA model that not all inefficiencies will be captured by the radial efficiency measures, but that slacks also have to be considered when judging the extent of inefficiency. In this model, efficiency relates to the observed ability to generate more output consuming less input (see the dual version of (4.1)). This model computes the distance between the efficient frontier, evaluated without unit $p$, and the unit itself. To highlight the practical implication of this model, consider the data set that consists of two inputs and one output.The data are listed in Table 4.1. 
Model 8 for evaluating $\mathrm{DMU}_{2}$ is as follows:

$$
\begin{gathered}
D_{2}=\operatorname{Max}-2 \alpha_{1}-2 \alpha_{2}+\beta \quad \text { subject to }-5 \alpha_{1}-\alpha_{2}+\beta+s_{1}=0, \\
-\alpha_{1}-4 \alpha_{2}+\beta+s_{2}=0, \\
\alpha_{1}+\alpha_{2}+\beta=1, \\
s_{1} \leq\left(1-\gamma_{1}\right) M, \\
s_{2} \leq\left(1-\gamma_{2}\right) M, \\
\gamma_{1}+\gamma_{2} \geq 2, \\
\gamma_{1}, \gamma_{2} \in\{0,1\} \cdot \alpha_{1}, \alpha_{2}, \beta \geq 0 .
\end{gathered}
$$

The optimal solution to this program is $\left(\alpha_{1}, \alpha_{2}, \beta\right)=(3 / 26,4 / 26,19 / 26), D_{2}=5 / 26$, and the supporting efficient surface is

$$
F^{\prime}=\left\{\left(x_{1}, x_{2}, y\right) \mid-\frac{3}{26} x_{1}-\frac{4}{26} x_{2}+\frac{19}{26} y=0\right\} \cup T_{c}^{\prime}
$$

(Note that $T_{c}^{\prime}$ is the new PPS obtained from omission of $\mathrm{DMU}_{p}$.) From (4.2) it is easy to show that $F^{\prime}=\left\{(X, Y):-\alpha^{*^{T}} X+\beta^{*^{T}} Y=0\right\} \cap T_{c}^{\prime}$ is an efficient supporting surface to $T_{c}^{\prime}$, in which $\left(\alpha^{*}, \beta^{*}\right)$ is the optimal solution to (4.1) in evaluating $\mathrm{DMU}_{2}$. Consider the following two cases:

(i) $F^{\prime}$ is unique and hence $\left(\alpha^{*}, \beta^{*}\right)$ is unique. In this case, set

$$
E F_{p}=\frac{D_{p}}{\sqrt{\alpha^{*^{T}} \alpha^{*}+\beta^{*^{T}} \beta^{*}}}
$$

(ii) $F^{\prime}$ is not unique. In this case, let $X_{\lambda}=\sum_{j \neq p} \lambda_{j} X_{j}, Y_{\lambda}=\sum_{j \neq p} \lambda_{j} Y_{j}$ in which $\lambda_{j} \geq 0$ for all $j$. Set

$$
E F_{p}=\operatorname{Min}_{\lambda \geq 0} \quad\left\{\text { the distance between }\left(X_{p}, Y_{p}\right) \text { and }\left(X_{\lambda}, Y_{\lambda}\right)\left(L_{2} \text {-norm }\right)\right\} .
$$

The proposed efficiency index is now defined as

$$
\widehat{E F}_{p}=\frac{E F_{p}}{\operatorname{Max}_{1 \leq j \leq n}\left\{E F_{j}\right\}} \leq 1 .
$$

It is to be noted that in a real data set, the second case is of seldom occurrence.

Definition 4.2. $\left(X_{p}, Y_{p}\right) \in T_{c}$ is nondominant if and only if there exist no $\lambda=\left(\lambda_{1}, \ldots\right.$, $\left.\lambda_{n}\right) \geq 0$ such that $\sum_{j=1}^{n} \lambda_{j} X_{j} \leq X_{p}$ and $\sum_{j=1}^{n} \lambda_{j} Y_{j} \geq Y_{p}$ and strict inequality holds true for at least one component.

The following theorem gives a necessary and sufficient condition for efficiency of $\mathrm{DMU}_{p}$.

Theorem 4.3. Considering (4.1), $D_{p} \geq 0$ if and only if $D M U_{p}$ is efficient in CCR model. 
Proof. We will first prove that if $D_{p} \geq 0$ then $\mathrm{DMU}_{p}$ is nondominant in $T_{c}$. Suppose, on the contrary, that $\mathrm{DMU}_{p}$ is dominant. Then, there exist some $\lambda=\left(\lambda_{1}, \ldots, \lambda_{n}\right)$ such that $\lambda_{j} \geq 0, j=1, \ldots, n$, and

$$
X_{p} \geq \sum_{j \neq p} \lambda_{j} X_{j}=X_{\lambda}, \quad Y_{p} \leq \sum_{j \neq p} \lambda_{j} Y_{j}=Y_{\lambda}
$$

and strict inequality holds true for at least one component. Now,

$$
0 \leq-\alpha^{*^{T}} X_{p}+\beta^{*^{T}} Y_{p}<-\alpha^{*^{T}} X_{\lambda}+\beta^{*^{T}} Y_{\lambda}
$$

where $\left(\alpha^{*}, \beta^{*}\right)$ is the optimal solution to (4.1). But we note that $\left(X_{\lambda}, Y_{\lambda}\right) \in T_{c}$ and

$$
H^{-}=\left\{(X, Y):-\alpha^{T} X+\beta^{T} Y \leq 0\right\}
$$

is a convex set, hence we must have

$$
-\alpha^{*^{T}} X_{\lambda}+\beta^{*^{T}} Y_{\lambda} \leq 0
$$

which contradicts (4.2). To show the converse, suppose that $\mathrm{DMU}_{p}$ is CCR-efficient; this means that $Z_{p}^{*} \geq 1$, where

$$
\begin{gathered}
Z_{p}^{*}=\operatorname{Max} U^{T} Y_{p}, \\
\text { s.t. } V^{T} X_{p}=1, \\
U^{T} Y_{j}-V^{T} X_{j} \leq 0, \quad j=1, \ldots, n, j \neq p, \\
U \geq \epsilon \cdot 1, \\
V \geq \epsilon \cdot 1 .
\end{gathered}
$$

Hence, if $\left(U^{*}, V^{*}\right)$ is the optimal solution of this problem, we must have $U^{T} Y_{p}-V^{T} X_{p}$ $\geq 0$. It is easy to show that $\left(U^{*}, V^{*}\right)$ is the optimal solution to this problem if and only if $\left(U^{*} /\left(1 U^{*}+1 V^{*}\right), V^{*} /\left(1 U^{*}+1 V^{*}\right)\right)=\left(\alpha^{*}, \beta^{*}\right)$ is the optimal solution to (3.3). Hence, we have

$$
\begin{gathered}
\sum_{r=1}^{s} \frac{u_{r}^{*}}{1 U^{*}+1 V^{*}} y_{r p}-\sum_{i=1}^{m} \frac{v_{i}^{*}}{1 U^{*}+1 V^{*}} x_{i p} \geq 0 \\
\Longrightarrow \beta^{* T} Y_{p}-\alpha^{* T} X_{p}=D_{p} \geq 0 .
\end{gathered}
$$

This completes the proof.

Hence, to rank DMUs, $\mathrm{DMU}_{j}$ is better than $\mathrm{DMU}_{i}$ if and only if $\widehat{E F}_{j}>\widehat{E F}_{i}$. In what follows, we show that the suggested efficiency index $\widehat{E F}_{j}$ is units-invariant. Suppose that $\left(X_{j}, Y_{j}\right), j=1, \ldots, n$, is substituted by $\left(\lambda X_{j}, \lambda Y_{j}\right)=\mathrm{DMU}_{\hat{j}}$, where $\lambda>0$. It suffices to show 
Table 4.2. The results for the simple example.

\begin{tabular}{cccc}
\hline Method & DMU $1_{1}$ & DMU $1_{2}$ & DMU $1_{3}$ \\
\hline CCR & 1.00 & 1.00 & 1.00 \\
Proposed method & 0.788 & 1.50 & 1.46 \\
AP method & 1.70 & Infeasible & 33.30 \\
\hline
\end{tabular}

Table 5.1. The data set for Example 5.1.

\begin{tabular}{ccccccc}
\hline & DMU1 & DMU2 & DMU3 & DMU 4 & DMU 5 & DMU 6 \\
\hline$I_{1}$ & 13 & 6 & 2 & 1 & 9 & 4 \\
$I_{2}$ & 1 & 3 & 6 & 10 & 5 & 8 \\
$\mathrm{O}$ & 1 & 1 & 1 & 1 & 1 & 1 \\
\hline
\end{tabular}

that $\widehat{E F}_{\hat{p}}=\widehat{E F}_{p}$. If $\left(\alpha^{*}, \beta^{*}\right)$ be the optimal solution to (4.1) for evaluation $\mathrm{DMU}_{p}$, it also is the optimal solution to (4.1) for evaluation $\mathrm{DMU}_{\hat{p}}$. Hence, $D_{\hat{p}}=\lambda D_{p}$. On the other hand,

$$
\begin{aligned}
& E F_{\hat{p}}=\frac{D_{\hat{p}}}{\sqrt{\alpha^{* T} \alpha^{*}+\beta^{*^{T}} \beta^{*}}}=\frac{\lambda D_{p}}{\sqrt{\alpha^{* T} \alpha^{*}+\beta^{*^{T}} \beta^{*}}}, \\
& \widehat{E F}_{\hat{p}}=\frac{E F_{\hat{p}}}{\operatorname{Max}_{1 \leq j \leq n}\left\{E F_{\hat{j}}\right\}}=\widehat{E F_{p}} .
\end{aligned}
$$

In this method, a small variation in data leads to a small shift on the frontier. Also, the proposed model is always feasible and bounded. These guarantee the feasibility and stability of the method. Consider the presented example in Section 3, again. Using the proposed method, we have the results presented in Table 4.2.

\section{Illustrative examples}

In order to provide a numerical illustration of the proposed approach, three examples are given.

Example 5.1. Consider the data set in Table 5.1 consisting of six DMUs each consuming two inputs to produce one output.

The results of the proposed approach are summarized in Table 5.2.

Example 5.2. Another example consists of a data set on 15 units with four inputs and three outputs. Table 5.3 contains a listing of the original data.

Running the proposed model yields the results listed in Table 5.4.

Example 5.3. In order to provide an application of the proposed ranking approach, the Sherman and Gold's [9] data set on 14 bank branches is used. Sherman and Gold utilized 
Table 5.2. The results for Example 5.1.

\begin{tabular}{cccccc}
\hline $\mathrm{DMU}_{j}$ & $\widehat{E F}_{j}$ & Classification & Proposed rank & AP rank & MAJ rank \\
\hline 1 & 1.000 & Efficient & 1 & 1 & 1 \\
2 & 0.339 & Efficient & 4 & 4 & 4 \\
3 & 0.454 & Efficient & 3 & 3 & 2 \\
4 & 0.726 & Efficient & 2 & 2 & 3 \\
5 & -1.122 & Inefficient & 6 & 6 & 6 \\
6 & -0.924 & Inefficient & 5 & 5 & 5 \\
\hline
\end{tabular}

Table 5.3. The raw data for Example 5.2.

\begin{tabular}{cccccccc}
\hline DMUs & $I_{1}$ & $I_{2}$ & $I_{3}$ & $I_{4}$ & $O_{1}$ & $O_{2}$ & $O_{3}$ \\
\hline 01 & 63.46 & 21.39 & 0 & 0 & 42.23 & 0 & 4063.68 \\
02 & 0 & 0 & 0 & 229.24 & 13.89 & 0 & 11250.82 \\
03 & 0 & 22.86 & 15.54 & 21.59 & 55.56 & 0 & 0 \\
04 & 199.62 & 5.47 & 0 & 52.29 & 56.17 & 0 & 9829.4 \\
05 & 302.82 & 15.52 & 0 & 0 & 92.59 & 0 & 0 \\
06 & 109.1 & 0 & 20.47 & 0 & 36.29 & 0 & 2410.96 \\
07 & 0 & 0 & 56.21 & 14.03 & 0 & 34.13 & 0 \\
08 & 6.38 & 11.52 & 43.03 & 0 & 15.14 & 13.04 & 10574.70 \\
09 & 33.61 & 28.34 & 0 & 75.24 & 69.46 & 0 & 4971.83 \\
10 & 167.28 & 0 & 55.79 & 0 & 0 & 29.87 & 12458.02 \\
11 & 99.64 & 9.77 & 6.76 & 0 & 0 & 19.89 & 10099.37 \\
12 & 0 & 23.71 & 0 & 0 & 0 & 27.78 & 0 \\
13 & 194.02 & 8.24 & 0 & 0 & 46.17 & 0 & 6506.39 \\
14 & 0 & 6.42 & 119.09 & 0 & 0 & 74.07 & 0 \\
15 & 0 & 10.83 & 7.31 & 10.23 & 26.32 & 0 & 0 \\
\hline
\end{tabular}

DEA to evaluate the technical efficiency of branch operation. The data set consists of three inputs and four outputs over 14 branches. The input and output categories are as follows:

Input 1: rent (thousands of dollars),

Input 2: full-time equivalent personnel, Input 3: supplies (thousands of dollars),

Output 1: loan applications, new pass-book loans, life-insurance sales,

Output 2: new accounts, closed accounts,

Output 3: travelers checks sold, bonds sold, bonds redeemed,

Output 4: deposits, withdrawals, checks sold, treasury checks issued, checks, loan payments, pass-book loan payments, life insurance payments, mortgage payments.

Table 5.5 contains a listing of the original data. 
Alireza Amirteimoori et al. 405

Table 5.4. The results for Example 5.2 using different algorithms.

\begin{tabular}{cccccc}
\hline $\mathrm{DMU}_{j}$ & $\widehat{E F}_{j}$ & Classification & Proposed rank & AP rank & MAJ rank \\
\hline 01 & 15.45 & Efficient & 6 & 4 & 6 \\
02 & 41.95 & Efficient & 2 & Infeasible & 3 \\
03 & 0.004 & Efficient & 15 & 10 & 15 \\
04 & 5.69 & Efficient & 12 & 7 & 12 \\
05 & 8.29 & Efficient & 9 & 8 & 10 \\
06 & 6.39 & Efficient & 11 & Infeasible & 9 \\
07 & 2.95 & Efficient & 13 & Infeasible & 13 \\
08 & 88.19 & Efficient & 1 & 1 & 1 \\
09 & 11.44 & Efficient & 7 & 5 & 7 \\
10 & 10.674 & Efficient & 8 & Infeasible & 8 \\
11 & 22.12 & Efficient & 5 & 6 & 5 \\
12 & 23.39 & Efficient & 3 & Infeasible & 2 \\
13 & 6.72 & Efficient & 10 & 3 & 11 \\
14 & 23.06 & Efficient & 4 & 2 & 4 \\
15 & 0.044 & Efficient & 14 & 9 & 14 \\
\hline
\end{tabular}

Table 5.5. The raw data for Example 5.3.

\begin{tabular}{crrrrrrc}
\hline DMUs & \multicolumn{1}{c}{$I_{1}$} & \multicolumn{1}{c}{$I_{2}$} & \multicolumn{1}{c}{$I_{3}$} & \multicolumn{1}{c}{$O_{1}$} & \multicolumn{1}{c}{$O_{2}$} & \multicolumn{1}{c}{$O_{3}$} & \multicolumn{1}{c}{$O_{4}$} \\
\hline 01 & 140,000 & 42,900 & 87,500 & 484,000 & $4,139,100$ & 59,860 & $2,951,430$ \\
02 & 48,800 & 17,400 & 37,900 & 384,000 & $1,685,500$ & 139,780 & $3,336,860$ \\
03 & 36,600 & 14,200 & 29,800 & 209,000 & $1,058,900$ & 65,720 & $3,570,050$ \\
04 & 47,100 & 9,300 & 26,800 & 157,000 & 879,400 & 27,340 & $2,081,350$ \\
05 & 32,600 & 4,600 & 19,600 & 46,000 & 370,900 & 18,920 & $1,069,100$ \\
06 & 50,800 & 8,300 & 18,900 & 272,000 & 667,400 & 34,750 & $2,660,040$ \\
07 & 40,800 & 7,500 & 20,400 & 53,000 & 465,700 & 20,240 & $1,800,250$ \\
08 & 31,900 & 9,200 & 21,400 & 250,000 & 642,700 & 43,280 & $2,296,740$ \\
09 & 36,400 & 76,000 & 21,000 & 407,000 & 647,700 & 32,360 & $1,981,930$ \\
10 & 25,700 & 7,900 & 19,000 & 72,000 & 402,500 & 19,930 & $2,284,910$ \\
11 & 44,500 & 8,700 & 21,700 & 105,000 & 482,400 & 49,320 & $2,245,160$ \\
12 & 42,300 & 8,900 & 25,800 & 94,000 & 511,000 & 26,950 & $2,303,000$ \\
13 & 40,600 & 5,500 & 19,400 & 84,000 & 287,400 & 34,940 & $1,141,750$ \\
14 & 76,100 & 11,900 & 32,800 & 199,000 & 694,600 & 67,160 & $3,338,390$ \\
\hline
\end{tabular}

Running AP, MAJ, and proposed models yield a ranking pattern which is reported in Table 5.6. Note that DMU1, DMU2, DMU3, DMU4, DMU6, DMU9, DMU10 and DMU14 are efficient in $T_{c}$. 
Table 5.6. The results for Example 5.3 using different algorithms.

\begin{tabular}{cccccc}
\hline $\mathrm{DMU}_{j}$ & $\widehat{E F}_{j}$ & Classification & Proposed rank & AP rank & MAJ rank \\
\hline 01 & 0.284 & Efficient & 4 & 6 & 4 \\
02 & 1.000 & Efficient & 1 & 2 & 1 \\
03 & 0.411 & Efficient & 3 & 4 & 3 \\
04 & 0.007 & Efficient & 8 & 8 & 8 \\
05 & -0.02 & Inefficient & 11 & 12 & 11 \\
06 & 0.263 & Efficient & 5 & 3 & 5 \\
07 & -0.105 & Inefficient & 14 & 14 & 14 \\
08 & -0.011 & Inefficient & 9 & 9 & 9 \\
09 & 0.737 & Efficient & 2 & 1 & 2 \\
10 & 0.046 & Efficient & 6 & 5 & 6 \\
11 & -0.016 & Inefficient & 10 & 10 & 10 \\
12 & -0.105 & Inefficient & 13 & 13 & 13 \\
13 & -0.026 & Inefficient & 12 & 11 & 12 \\
14 & 0.011 & Efficient & 7 & 7 & 7 \\
\hline
\end{tabular}

\section{Conclusion}

In multidimensional cases, or if the number of observations is relatively small as compared to the sum of number of inputs and outputs, running the DEA model will typically result in a relatively large number of efficient units. Often, decision makers are interested in a complete ranking, beyond the dichotomized classification, in order to refine the evaluation of the units. This paper provides an analytical procedure for ranking efficient units. In this method, the difficulties of the existing methods have been removed. The proposed approach can be analytically separated in the following two computational processes: (i) the first stage omits the unit being scored from PPS and determines the new frontier and (ii) the second stage finds the shortest distance from the unit being scored to new frontier.

\section{References}

[1] P. Andersen and N. C. Petersen, A procedure for ranking efficient units in data envelopment analysis, Management Sci. 39 (1993), no. 10, 1261-1264.

[2] T. R. Anderson, K. Hollingsworth, and L. Inman, The fixed weighting nature of a crossevaluation model, Manag. Sci. 17 (2002), no. 3, 249-255.

[3] R. D. Banker, A. Charnes, and W. W. Cooper, Some models for estimating technical and scale inefficiencies in data envelopment analysis, Management Sci. 30 (1984), no. 9, 1078-1092.

[4] A. Charnes and W. W. Cooper, Programming with linear fractional functionals, Naval Res. Logist. Quart. 9 (1962), 181-186.

[5] A. Charnes, W. W. Cooper, and E. Rhodes, Measuring the efficiency of decision making units, European J. Oper. Res. 2 (1978), no. 6, 429-444. 
[6] W. D. Cook, M. Kress, and L. M. Seiford, Prioritization models for frontier decision making units in DEA, Eur. J. Oper. Res. 59 (1992), no. 2, 319-323.

[7] M. Farell, The measurement of productive efficiency, J. Roy. Statist. Soc. Ser. A 120 (1957), 253281.

[8] S. Mehrabian, M. R. Alirezaee, and G. R. Jahanshahloo, A complete efficiency ranking of decision making units in data envelopment analysis, Comput. Optim. Appl. 14 (1999), no. 2, 261266.

[9] H. D. Sherman and F. Gold, Bank branch operating efficiency: evaluation with DEA, J. Bank. Fin. 9 (1985), 297-315.

Alireza Amirteimoori: Department of Mathematics, Islamic Azad University, P.O. Box 41335-3516, Rasht, Iran

E-mail address: teimoori@guilan.ac.ir

Gholamreza Jahanshahloo: Department of Mathematics, Teacher Training University, P.O. Box 1316, Tehran, Iran

E-mail address: guilanmath@iaurasht.ac.ir

Sohrab Kordrostami: Department of Mathematics, Islamic Azad University, P.O. Box 1616, Lahijan, Iran

E-mail address: krostami@guilan.ac.ir 


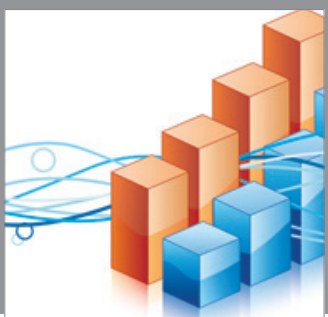

Advances in

Operations Research

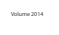

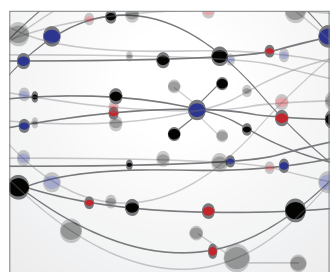

\section{The Scientific} World Journal
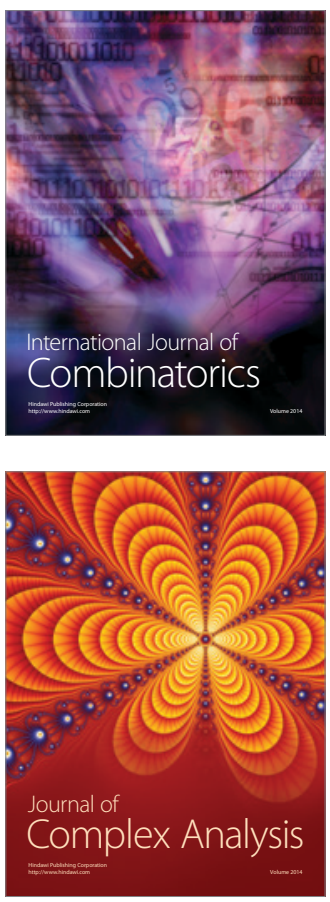

International Journal of

Mathematics and

Mathematical

Sciences
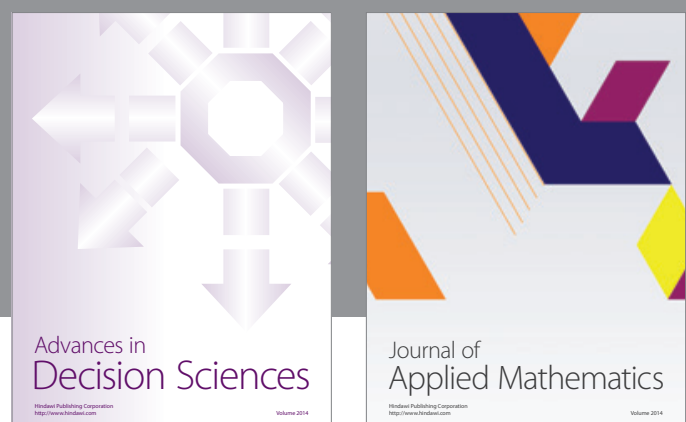

Journal of

Applied Mathematics
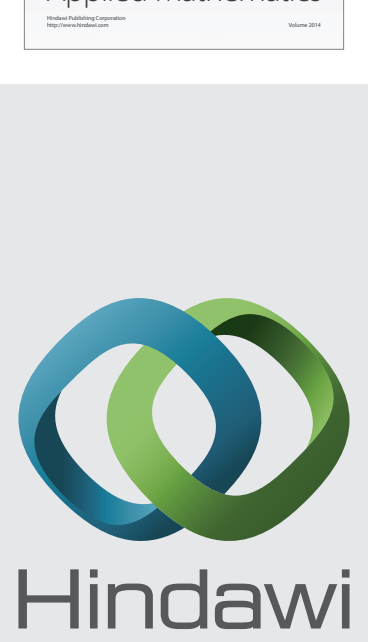

Submit your manuscripts at http://www.hindawi.com
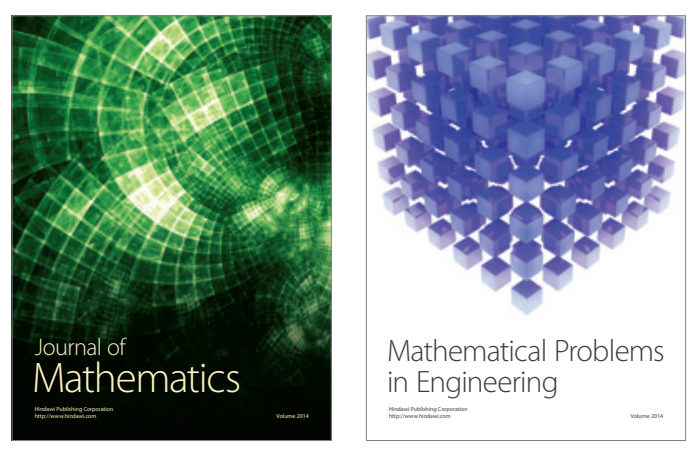

Mathematical Problems in Engineering
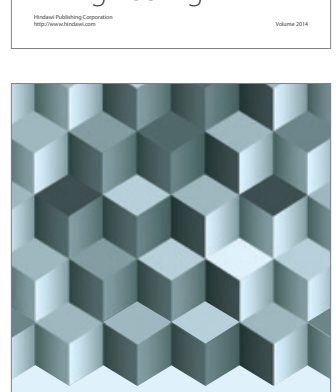

Journal of

Function Spaces
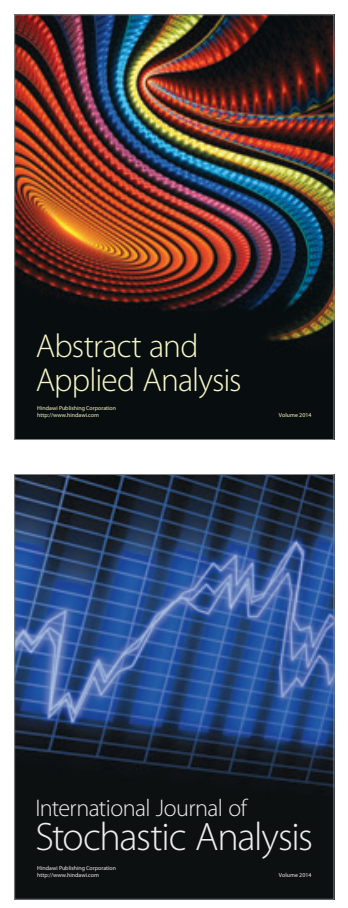

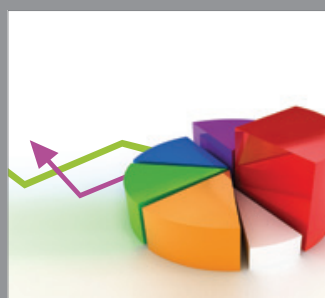

ournal of

Probability and Statistics

Promensencen
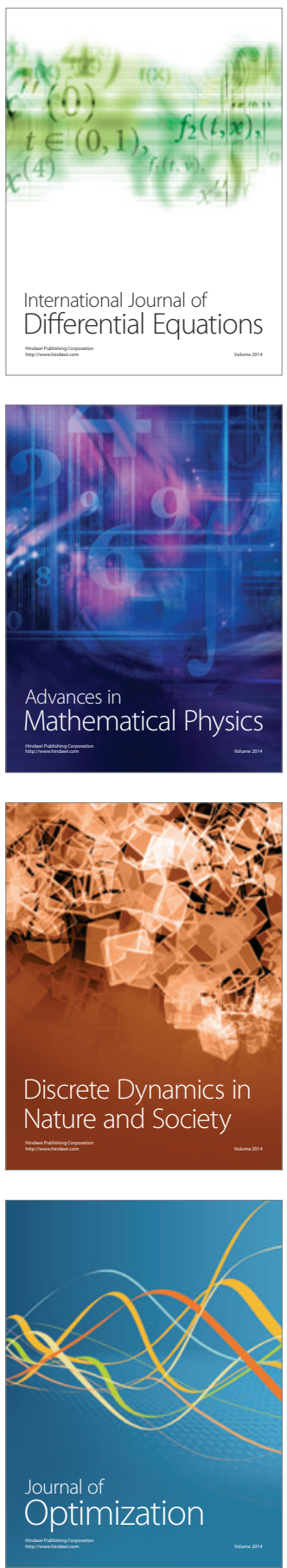